\title{
Synthesis of single-crystal hyperbranched rhodium nanoplates with remarkable catalytic properties
}

\author{
Jiawei Zhang, Meishan Chen, Jiayu Chen, Huiqi Li, Suheng Wang, Qin Kuang*, Zhenming Cao and \\ Zhaoxiong Xie*
}

\begin{abstract}
The catalytic properties of noble metal nanocrystals can be tuned via engineering their structures. Nanocrystals with fractal structures are fascinating catalysts regarding their large surface area-to-volume ratios, large numbers of edges and corners, which can be tuned simultaneously by their hierarchical ordering. However, it is still a great challenge to control the hierarchical ordering of noble metal fractal nanocrystals and their formation mechanism is not fully understood. Herein, we report a facile solvothermal method for the direct preparation of a unique single-crystal Rh-hyperbranched structure, which consists of hierarchically ultrathin nanoplates with threefold symmetry, large surface area and high density of low-coordinated edge/corner sites. Importantly, the hierarchical ordering can be readily tuned by changing the composition of solvent. In addition, we found the as-prepared single-crystal hyperbranched $\mathrm{Rh}$ nanoplates possessed great structure stability, and exhibited better catalytic performance towards both ethanol electrooxidation and hydrogenation of styrene than the commercial Rh black, which can be attributed to the large surface area and high-dentisty of edge/corner sites.
\end{abstract}

Keywords: rhodium, fractal growth, nanostructures, electrocatalysis, hydrogenation

\section{INTRODUCTION}

Noble metal nanocrystals (NCs) have received increasing interest owing to their indispensable role in energy-related catalytic fields [1-6]. The scarce reserves and high cost of noble metals make it imperative to improve the atom utilization efficiencies and the performances of noble metal-based catalysts. In the past decades, tremendous efforts have been devoted to improve the catalytic properties of noble metal-based materials mostly by tailoring the sizes, shapes, and surface structures of the noble metal NCs having well-defined polyhedral shapes [7-11]. For example, upon decreasing the size of NCs, the specific surface area and the fraction of unsaturated surface atoms dramatically increase, thereby improving the catalytic reactivity and atom utilization efficiency values [12]. By tailoring the shape of NCs, the exposed surface structure can be tuned and optimized, thereby leading to highly active and selective catalytic sites [13]. Recently, it was found through preparing some specific morphologies, such as nanoframes and jagged nanowires, high density of edge/coner atoms and superior catalytic performances could be directly generated [14,15]. In this sense, noble metal NCs with fractal structures, characterized by a high density of low-coordinated edge/corner sites and large surface-to-volume ratios, would be also promising catalysts.

Fractal structures (e.g., snowflakes and long coastlines) characterized by self-similar forms with multi-order branching are ubiquitous in nature [16-19]. In fact, these structures can potentially present infinite boundaries in a limited space. For instance, according to the famous mathematical Koch snowflake pattern, the area of the snowflake only increases to 1.6 times as compared to the original equilateral triangle, while its perimeter approaches to infinity [20]. In nanoscience, the ability to control the hierarchical ordering of fractal structures allows simultaneously tuning the number of low-coordinated edge/corner sites and the surface-to-volume ratios, two key factors governing the catalytic properties of noble metal NCs. However, it is thermodynamically unfavored for noble metals to grow into fractal structures as they tend to form convex polyhedral shapes to minimize the surface free energy during crystal growth. De-

State Key Laboratory for Physical Chemistry of Solid Surfaces, Collaborative Innovation Center of Chemistry for Energy Materials, Department of Chemistry, College of Chemistry and Chemical Engineering Xiamen University, Xiamen 361005, China

* Corresponding authors (emails: qkuang@xmu.edu.cn (Kuang Q); zxxie@xmu.edu.cn (Xie Z)) 
spite some progresses made in the preparation of fractallike structures (e.g., dendritic Ag NCs, tri/multi-pod Au, $\mathrm{Pt}$ and Pd NCs, etc.), there is still no effective approach to control the fractal structures, especially their hierarchical ordering [21-27].

On the other hand, $\mathrm{Rh}$ is an important functional material with excellent catalytic performances in a wide variety of important applications such as hydrogenation, hydroformylation, $\mathrm{CO}$ oxidation, $\mathrm{NO}_{x}$ reduction, and fuel cell-related reactions [28-31]. In the last decade, great efforts have been made to improve the catalytic properties of this noble metal by tailoring the morphologies and surface structures of Rh NCs [32-40]. However, owing to the extraordinarily high surface free energy of Rh, successful approaches leading to Rh NCs with high density of low-coordinated edge/corner sites or large surface-tovolume ratios have been scarcely described in the literature. In this paper, we realized the controlled synthesis of a peculiar form of hyperbranched single-crystal Rh fractal nanostructures via a facile solvothermal method. Importantly, the hierarchical ordering of these structures along with the density of low-coordinated edge/corner sites and the surface-to-volume ratios can be readily tuned. Our experimental results demonstrate that the hyperbranched fractal $\mathrm{Rh}$ nanostructures prepared herein possess a great structural stability while exhibiting significantly enhanced electrocatalytic and hydrogenation activities as compared to a commercial Rh black catalyst. The large surface area and high density of edge/corner sites can account for the superior catalytic activities of these materials.

\section{EXPERIMENTAL SECTION}

\section{Chemicals}

Rhodium(III) chloride hydrate $\left(\mathrm{RhCl}_{3} \cdot x \mathrm{H}_{2} \mathrm{O}, 38 \%-41 \%\right.$ $\mathrm{Rh})$ was purchased from J\&K Scientific Ltd.; 1-octadecylamine (ODA, 97\%) and Rh black were purchased from Alfa Aesar, tert-butylamine (TBA, 99.5\%), chloroform $\left(\mathrm{CHCl}_{3}, \mathrm{AR}\right)$, perchloric acid $\left(\mathrm{HClO}_{4}, \mathrm{AR}\right)$, sodium hydroxide $(\mathrm{NaOH}, \mathrm{AR})$, and ethanol $\left(\mathrm{C}_{2} \mathrm{H}_{5} \mathrm{OH}, \mathrm{AR}\right)$ were purchased from Sinopharm Chemical Reagent Co. Ltd. (Shanghai, China). Hydrogen $\left(\mathrm{H}_{2}, 99.999 \%\right)$, nitrogen $\left(\mathrm{N}_{2}\right.$, 99.99\%) and mixed gas $\left(4 \% \mathrm{CO}+96 \% \mathrm{~N}_{2}\right)$ were purchased from Linde Industrial Gases. All reagents were used as received without further purification.

\section{Synthesis of hyperbranched $\mathrm{Rh}$ triangle nanoplates}

In a typical synthesis, $\mathrm{RhCl}_{3} \cdot x \mathrm{H}_{2} \mathrm{O}(5.0 \mathrm{mg})$ was mixed with $8 \mathrm{~mL}$ ODA. The resulting homogeneous yellow so- lution was transferred to a $25-\mathrm{mL}$ Teflon-lined stainlesssteel autoclave. The sealed vessel was then heated at $200^{\circ} \mathrm{C}$ for $10 \mathrm{~h}$ before it was cooled to $70^{\circ} \mathrm{C}$. The black products were separated via centrifugation and further purified by an ethanol-chloroform (1:4) mixture several times for further use. For tailoring the sizes, the ODA was replaced by equivalent volume of TBA while keeping other conditions unchanged. For convenience, the as-synthesized $\mathrm{Rh}$ samples are referred to herein as $\mathrm{Rh}$ branch- $(8-x): x$, where $x$ represents the volume of TBA in the growth solutions.

\section{Characterizations}

The morphology and crystal structure of the as-prepared products were observed by scanning electron microscopy (SEM, Hitachi S4800) and high-resolution transmission electron microscopy (HRTEM, JEM 2100) with an acceleration voltage of $200 \mathrm{kV}$. The samples were prepared by dropping chloroform dispersion of samples onto carbon-coated copper TEM grids. The phases of the asprepared products were determined by powder X-ray diffraction (XRD) pattern using a Rigaku Ultima IV X-ray diffractometer with $\mathrm{Cu} \mathrm{Ka}$ radiation. Diffuse reflectance infrared Fourier transform spectroscopy (DRIFTS) was conducted on a Nicolet 6700 spectrometer by depositing the chloroform suspensions of hyperbranched Rh NCs on dried $\mathrm{KBr}$ powders followed by solvent evaporation.

\section{Box-counting estimation of the fractal dimension}

In this method, square boxes with a size of $\varepsilon$ were used to cover the pattern. $N(\varepsilon)$ represented the number of boxes required to cover the dendritic pattern. The box-counting dimension can be defined as:

$$
D_{B}=\lim _{\varepsilon \rightarrow 0} \log N(\varepsilon) / \log (1 / \varepsilon) .
$$

\section{Pre-treatment of the hyperbranched Rh nanoplates before} catalytic applications

Before catalytic tests, a pre-treatment based on ligand exchanging was conducted to clean the surface of catalysts. In a typical process, a batch of as-prepared branched $\mathrm{Rh}$ NCs was stirred with $10 \mathrm{~mL} n$-butylamine for $72 \mathrm{~h}$ at room temperature. After that, the hyperbranched $\mathrm{Rh}$ nanoplates were collected by centrifugation at 10,000 rpm for $8 \mathrm{~min}$ and washed three times with ethanol to remove the excess surface adsorbed amines. The product was dried at $65^{\circ} \mathrm{C}$ in vacuum oven overnight for further catalytic measurements.

\section{Electrocatalytic measurement}

A glassy carbon electrode (diameter $5 \mathrm{~mm}$ ) was carefully 
polished and washed before each experiment. The dried hyperbranched $\mathrm{Rh}$ triangle nanoplates were dispersed in chloroform to get a $1.0 \mathrm{mg} \mathrm{mL}^{-1}$ ink. In a typical experiment, $5.0 \mu \mathrm{L}$ of the suspensions were deposited on a glassy carbon electrode, which was used as the working electrode after the solvent volatilized. A Pt slice and a saturated calomel electrode (SCE) served as the counter electrode and the reference electrode, respectively. Before measurement, the working electrode was electrochemically cleaned by continuous potential cycling between -0.35 and $1.00 \mathrm{~V}$ at $50 \mathrm{mV} \mathrm{s}^{-1}$ in $\mathrm{N}_{2}$-saturated 0.1 mol L ${ }^{-1} \mathrm{HClO}_{4}$ solution until a stable cyclic $\mathrm{CV}$ curve was obtained.

For the CO-stripping voltammetry measurements, $\mathrm{CO}$ gas was bubbled for $15 \mathrm{~min}$ through the $0.1 \mathrm{~mol} \mathrm{~L}^{-1}$ $\mathrm{HClO}_{4}$ solution in which the electrode was immersed. After that, $\mathrm{N}_{2}$ was bubbled for additional 5 min to remove the $\mathrm{CO}$ in the solution. The CO-stripping cyclic voltammetry was recorded from -0.25 to $1.00 \mathrm{~V}$ with a scan rate of $20 \mathrm{mV} \mathrm{s}^{-1}$.

The catalytic activity was measured by cyclic voltammetry method in a solution containing $1.0 \mathrm{~mol} \mathrm{~L}^{-1} \mathrm{NaOH}$ $+1.0 \mathrm{~mol} \mathrm{~L}^{-1} \mathrm{C}_{2} \mathrm{H}_{5} \mathrm{OH}$ with a scan rate of $50 \mathrm{mV} \mathrm{s}^{-1}$, while the $\mathrm{Hg} / \mathrm{HgO}\left(1 \mathrm{~mol} \mathrm{~L}^{-1} \mathrm{KOH}\right)$ was served as the reference electrode. The electrochemically active surface area (ECSA) was determined according to the equation ECSA $=Q / m q_{0}$, where the $Q$ is the electric charge calculated from the area of the $\mathrm{CO}$ absorption peaks at $26^{\circ} \mathrm{C}$ in $\mathrm{N}_{2}$ saturated $0.1 \mathrm{~mol} \mathrm{~L}^{-1} \mathrm{HClO}_{4}$ solution, where $q_{0}$ is 380 $\mu \mathrm{C} \mathrm{cm}^{-2}, m$ is the loading mass of catalysts on the working electrode.

\section{Hydrogenation of styrene}

In a typical catalytic reaction, the suspension of $8.0 \mathrm{~mL}$ chloroform, $4.0 \mathrm{~mL}$ ethanol, $228.6 \mu \mathrm{L}$ styrene $\left(2 \mathrm{mmol} \mathrm{L}^{-1}\right)$ and $0.4 \mathrm{mg}$ of Rh catalysts $\left(0.004 \mathrm{mmol} \mathrm{L}^{-1}\right)$ was placed into a glass pressure vessel with $75 \mathrm{~mL}$ of capacity. To remove air from the reaction system, the autoclave was purged with $\mathrm{H}_{2}$ under pressure of $0.2 \mathrm{MPa}$ for several minutes. Subsequently, the autoclave was pressurized with $0.2 \mathrm{MPa}_{2}$ and stirred magnetically at $30^{\circ} \mathrm{C}$ for $1 \mathrm{~h}$. The reaction was stopped every 15 minutes to extract $200 \mu \mathrm{L}$ solution for gas chromatographic (GC2014C, Shimadzu Corporation) analysis and conversion determination.

\section{RESULTS AND DISCUSSION}

The single-crystal hyperbranched $\mathrm{Rh}$ fractal nanostructures were prepared via a facile solvothermal route at $200^{\circ} \mathrm{C}$ for $10 \mathrm{~h}$ by using $\mathrm{RhCl}_{3}(5 \mathrm{mg})$ as a precursor and octadecylamine (ODA, $8 \mathrm{~mL}$ ) as both solvent and reducing agent in the absence of any other surfactant or substrate. Fig. 1a shows the powder XRD pattern of the as-prepared sample. All the diffraction peaks were well indexed to a $f c c$ metallic Rh structure (JCPDS No.050685). The broadening of the diffraction peaks was attributed to the ultrathin plate-like structural feature of the sample. The TEM images shown in Fig. 1b, $c$ and the SEM image in Fig. S1 clearly reveal the presence of highly ordered hyperbranched $\mathrm{Rh}$ nanoplates with a well-defined triangle outline. This outline shows three main arms rotated by $120^{\circ}$ with respect to each other and an arm length $a$ (i.e., the distance from the center to the vertex) of $81.3 \pm$ $8.8 \mathrm{~nm}$. In essence, these triangle nanoplates with multiple-order branching can be considered as highly ordered assemblies built with numerous primary triangles growing each other from the three vertices of a triangle, as illustrated in Fig. 1b. Electron diffraction (ED) characterization further reveals that these hyperbranched $\mathrm{Rh}$ triangle nanoplates are single-crystalline, with the top/ bottom surfaces being bounded by $\{111\}$ planes, and the three main arms growing along the three $\langle 211\rangle$ directions of the $f c c$ Rh structure. Remarkably, the appearance of weak 1/3(422) diffraction spots in the ED pattern (marked as a triangle in Fig. 1c, inset), normally forbidden for $f c c$ structured metals, indicates that the as-prepared hyperbranched $\mathrm{Rh}$ triangle nanoplates contain high-density stacking faults or twin planes parallel to (111) planes [41]. The perfect single-crystalline nature of such hyperbranched $\mathrm{Rh}$ triangle nanoplates was also confirmed by the corresponding HRTEM image (Fig. 1d), are the lattice fringes with an interplanar spacing of $0.23 \mathrm{~nm}$, corresponding to the 1/3(422) fringes of $f c c$ Rh (Fig. 1d) are aligned across the entire branch. The dark-field microscopic images (Fig. 1e, f) reveal no bright strain fringes near the junction zone between the two triangles, thereby indicating the absence of defects. With the aim to obtain the thickness information, vertically oriented hyperbranched $\mathrm{Rh}$ triangle nanoplates are specifically characterized (Fig. S2). Based on the analysis of more than 60 $\mathrm{Rh}$ triangle nanoplates, the thickness is estimated to be 4.0 $\pm 0.7 \mathrm{~nm}$ (Fig. 1g, h).

Morphologically, these as-prepared $\mathrm{Rh}$ fractal NCs possess a highly ordered hyperbranched feature, which is completely different from those previously reported planar tri- or multi-pods. The latter shows only a one-order branched morphology with three- or multi-arms. The materials prepared herein are also different from the dendritic structures that are usually disorderly attached self-assemblies of irregular primary units (Table S1). In 

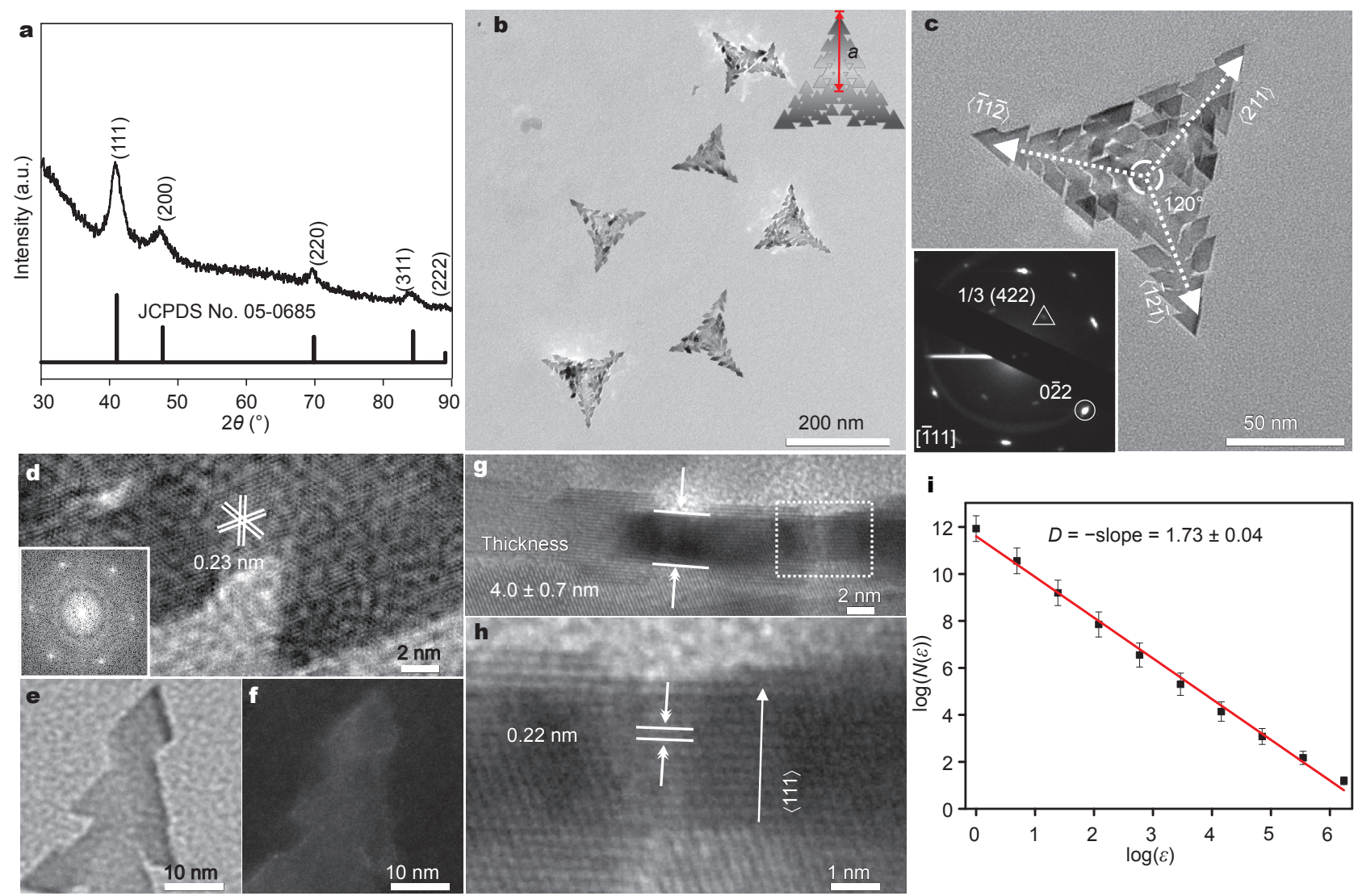

Figure 1 (a) Typical powder XRD pattern and (b) low-magnification TEM image of the as-prepared hyperbranched Rh triangle nanoplates. Inset in (b) shows the corresponding ideal model of the hyperbranched structure. (c) TEM image of an individual Rh NC projected from the [111] direction. The inset corresponds to the selected area electron diffraction pattern. The bright spots (circle) correspond to the $\{220\}$ Bragg reflections, and the weak spots (triangle) closer to the center are attributed to $1 / 3\{422\}$ forbidden reflections. (d) HRTEM image and corresponding fast-Fourier-transform pattern near the junction zone. (e) Bright-field and (f) corresponding dark-field TEM image of the tip of the hyperbranched Rh triangle nanoplates. (g) TEM image of hyperbranched Rh triangle nanoplates viewed from the side, and (h) zoom-in view of the area boxed in (g). (i) Box-counting estimation of the fractal dimension for the as-prepared hyperbranched $\mathrm{Rh}$ triangle nanoplates.

the case of the fractal structures, the fractal dimension $(D)$ can serve as a self-similarity measure of the structure over multiple scales of dimension. In the case of two-dimensional fractal structures, $D$ is a non-integer value between 1 (for line) and 2 (for plane) [16,42]. According to the result estimated by the box counting method (see Supplementary information for details) shown in Fig. 1i, the hyperbranched $\mathrm{Rh}$ triangle nanoplates show a $D$ value of $1.73 \pm 0.04$, significantly lower than 2 that is typically for a compact triangle.

The formation of highly symmetrical hyperbranched Rh NCs is interesting. To gain insight into the formation mechanism, a time-dependent experiment was conducted. Fig. 2a-d show representative TEM images of the growth intermediates collected at different reaction times. No products could be collected within $1 \mathrm{~h}$. The products collected at $2 \mathrm{~h}$ were dominated by distributed triangle nanoplates with a side length of $25 \pm 5 \mathrm{~nm}$, together with a few branched materials with three arms (Fig. 2a). This indicates that the triangle nanoplates are building blocks for those three-armed branched nanoplates. As the reaction progressed $(3 \mathrm{~h})$, second and even third order branching was developed in the primary triangle nanoplates and the arm length quickly grew (Fig. 2b). The branched nanoplates maintain the threefold symmetry during the growth. After continued growth up for $5 \mathrm{~h}$, almost all the $\mathrm{Rh}$ triangle nanoplates in the products show a hyperbranched structure (Fig. 2c). The morphology and size of the hyperbranched Rh NCs remained nearly unchanged for reaction times up to $10 \mathrm{~h}$ (Fig. 2d). It should be noted that except for the initial reaction stages, few distributed triangle nanoplates (i.e., building blocks for the hyperbranched structure) were produced in subsequent reaction periods. Thus, the fractal growth of the 


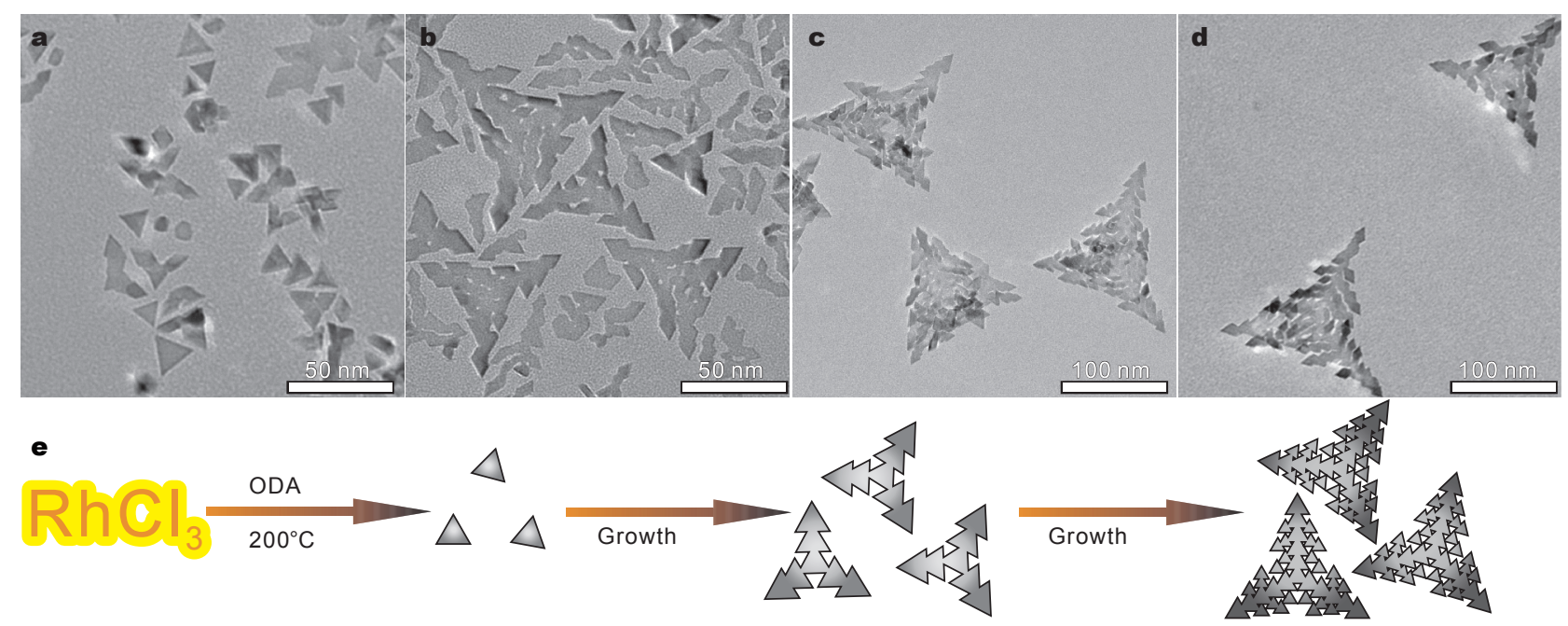

Figure 2 Representative TEM images of the hyperbranched Rh triangle nanoplates obtained at different reaction times: (a) $2 \mathrm{~h}$, (b) $3 \mathrm{~h}$, (c) $5 \mathrm{~h}$, and (d) $10 \mathrm{~h}$, showing the evolution of morphology with time. (e) Schematic illustration for the growth of the hyperbranched Rh triangle nanoplates.

highly ordered hyperbranched Rh triangle nanoplates did not follow the oriented attachment mechanism often invoked to explain the formation of single-crystalline branched or hierarchical nanostructures [43-45]. Based on the above observation, we deduced that the highly ordered hyperbranched $\mathrm{Rh}$ triangle nanoplates resulted from the continuous growth of primary triangle nanoplate seeds, as illustrated in Fig. 2e.

Randomly dendritic structures are usually formed by growing branched structures that strictly obey a diffusionlimited aggregation mechanism $[46,47]$. The highly symmetrical fractal structures reported herein implied it followed a more complex growth mechanism as compared to a simple diffusion-limited growth. In our experiments, the sealed autoclave provided a high reaction temperature and high pressure environment for growth. Such unstable and oscillating experimental conditions may stimulate new nucleus to appear at the tips of the existing seeds, where the growth is significantly favored because of the advantageous location for obtaining the crystal-grown precursors [48-50]. The successive nucleation process followed the crystallographic orientation related to the crystal anisotropy of the noble metal. Thus, a fractal structure with anisotropy was obtained during the diffusion-limited growth process, which was defined as the nucleation-limited aggregation growth [48-50].

For the fractal structures under diffusion-limited growth conditions, the structure was very sensitive to the diffusion of the growth units. Theoretically speaking, the diffusion of the growth units in the growth solution can be expressed by the Fick's first law as follows:

$$
J=-D \frac{\mathrm{d} c}{\mathrm{~d} x}
$$

where $J$ is the diffusion flux of the growth units, $\frac{\mathrm{d} c}{\mathrm{~d} x}$ is the concentration gradient, and $D$ is the diffusion coefficient. Furthermore, $D$ can be determined with the Stokes-Einstein relation:

$$
D=\frac{R T}{6 \pi \eta r N_{A}},
$$

where $\eta$ is the viscosity of the solvent, $r$ is the radius of the atoms diffusing in the solvent, $N_{\mathrm{A}}$ is the Avogadro constant, $T$ is the absolute temperature, and $R$ is the gas constant [51]. Therefore, in a wet-chemical reduction environment at a given reaction temperature, the diffusion of the growth units can be adjusted by controlling their concentration and the viscosity of the solvent.

The viscosity directly affects the diffusion coefficient of the growth units. In this regard, the composition of the growth solution (especially the solvent) plays a vital role in forming branched structures. In our system, the ODA that contains a long $n$-alkyl chain was used as both solvent and reducing agent. It is well known that the molecules containing $n$-alkyl chain are easy to interdigitate each other, and the intermolecular crosslinking leads to the high viscosity of the growth solution [52-54]. Besides, the large steric barrier coming from the $n$-alkyl chain of ODA between the surface of the crystal nucleus and the bulk solution may further limit the diffusion of the growth 
units. These two main effects (i.e., high viscosity and large steric barrier) provided the solvent with a low diffusion coefficient for the growth units, thereby favoring the formation of the hyperbranched structure. As recently described by Peng and co-workers [55], utilization of branched organic molecules can significantly reduce the steric barrier. Thus, it is reasonable that the diffusion rate of growth units could be adjusted by introducing certain solvents with branched structure, thereby leading to the morphology evolution for the hyperbranched structures. In this sense, we gradually introduced TBA into the growth solution by replacing ODA with equivalent volumes of TBA. Different ODA to TBA volume ratios were used (i.e., $8 \mathrm{~mL}: 0 \mathrm{~mL}, 6 \mathrm{~mL}: 2 \mathrm{~mL}$, and $4 \mathrm{~mL}: 4 \mathrm{~mL}$ ). For convenience, the as-synthesized fractal $\mathrm{Rh}$ nanoplates were referred herein as $\mathrm{Rh}$ branch- $(8-x): x$, where $x$ represents the volume of TBA in the growth solutions. As shown in Fig. 3a-c (see Fig. S3 for low-magnification TEM images), the Rh samples are still dominated by hyperbranched $\mathrm{Rh}$ triangle nanoplates at the different ODA to TBA ratios studied herein (8:0, 6:2, and 4:4). Notably, the arm length of these hyperbranched Rh triangle nanoplates gradually decreased with the increasing volume of TBA in the growth solution $(81.3 \pm 8.8,52.7 \pm 4.9$, and $26.8 \pm 2.9 \mathrm{~nm}$ for the $8: 0,6: 2$, and 4:4 ODA to TBA ratios, respectively, black line in Fig. $3 \mathrm{~d}$ ). At the same time, the hierarchical ordering decreased with the progressively increasing volume of TBA, and a tri-pod structure was formed (Fig. 3c). The corresponding fractal dimensions slightly decreased with the volume of TBA $(1.73 \pm 0.04$, $1.67 \pm 0.05$, and $1.62 \pm 0.07$ for the Rh branch-8:0, Rh branch-6:2, and Rh branch-4:4 samples, respectively, red line in Fig. 3d and Fig. S4). The larger the fractal dimension $D$ is, the more compact a branched structure is. Here, along with the branched patterns getting less compact, the hierarchical ordering decreased, as well as the fractal dimensions and the sizes. However, when only TBA was used in the growth solution, irregular Rh NCs with unbranched structures were produced (Fig. S5). This result indicates that the crystal growth of Rh NCs in pure TBA is beyond the scope of a diffusion-limited growth. Besides, the effect of the reaction temperature was also investigated. There was no product below $150^{\circ} \mathrm{C}$. At $160^{\circ} \mathrm{C}$, the obtained NCs were still fractal nanostructures although severe aggregation occurred (Fig. S6a). After the reaction temperature was elevated to $220^{\circ} \mathrm{C}$, the as-prepared nanocrystals were still hyperbranched $\mathrm{Rh}$ nanoplates (Fig. S6b). This minor change may be due to the limited viscosity disturbance by varying the reaction temperature in our synthetic system.
In order to further investigate the role of ODA and TBA in the hierarchical ordering change process, the asprepared samples and pure ODA and TBA were analyzed by DRIFTS (Fig. 3e, f). The triplets at 2958, 2920, and $2852 \mathrm{~cm}^{-1}$ were ascribed to the $\mathrm{CH}_{2}$ asymmetric and symmetric stretching modes of the ODA chain, while the band at $720 \mathrm{~cm}^{-1}$ was produced by the $-\left(\mathrm{CH}_{2}\right)_{n^{-}}(n>3)$ rocking vibration [56]. The peaks located at ca. 1240 and $1370 \mathrm{~cm}^{-1}$ were assigned to the $t$-butyl groups and to the symmetric $\mathrm{CH}_{3}$ deformation vibration of the $t$-butyl groups, respectively [56]. These IR studies revealed that, despite using two solvents, only ODA was present in all the prepared hyperbranched $\mathrm{Rh}$ triangle nanoplates. As shown in Fig. 3f, when comparing the spectra of pure ODA and the hyperbranched Rh triangle nanoplates with different sizes, a series of peaks were found in the $1350-1180 \mathrm{~cm}^{-1}$ region which were ascribed to the all trans configuration of the $-\mathrm{CH}_{2}$ - groups in the hydrocarbon chain [55]. However, these peaks were less intense while increasing the volume of TBA, thereby revealing a progressive interruption of the trans configuration. These IR results suggested that ODA can be strongly adsorbed on the Rh surfaces, while TBA may mainly act as the ligand regulator. The interdigitated pack formed by ODA would be destroyed by the branched TBA, and the reduced steric barrier between the surface of NCs and the bulk solution would accelerate the diffusion rate of the growth units. Besides the steric effect, TBA also reduced the overall viscosity by destroying the intermolecular crosslinking of ODA, thereby accelerating the diffusion of the growth units. Overall, the diffusion rate of the growth units can be deliberately accelerated by progressively increasing the volume of TBA. Once the diffusion of the growth units no longer governed the crystal growth process, the hyperbranched structure disappeared.

As indicated in Equation (1), the concentration gradient has a significant effect on the diffusion flux. In this study, the concentration gradient originated from the different concentrations of precursor near the surface of the crystal nucleus and in the bulk solution. Therefore, the influence of the precursor concentration was also investigated, and the results are shown in Fig. S6. When only $0.25 \mathrm{mg} \mathrm{RhCl}_{3}$ were added (5\% as compared to the standard synthesis), flower-like assemblies consisting of nanoplates and small nanoparticles were formed (Fig. S7a). At such low concentration, there is no sufficient growth units to diffuse after the nucleation, thereby avoiding the formation of branched NCs. As the concentration of the precursor increased, the thickness of the diffusion layer decreased that in turn increased the concentration gradient, thus 
a

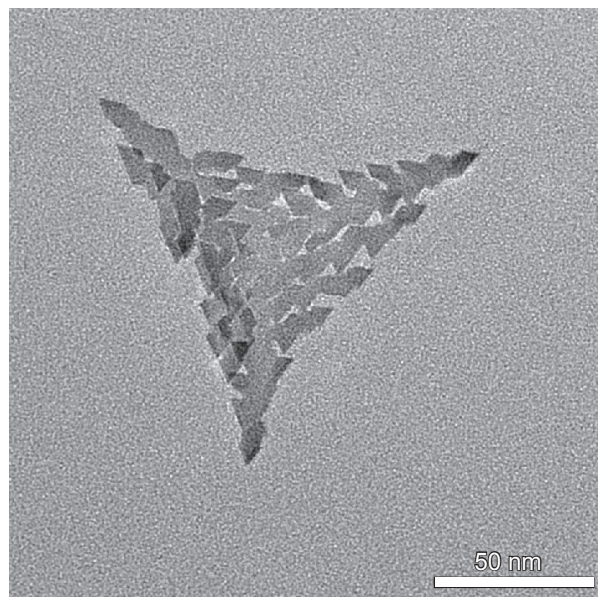

c
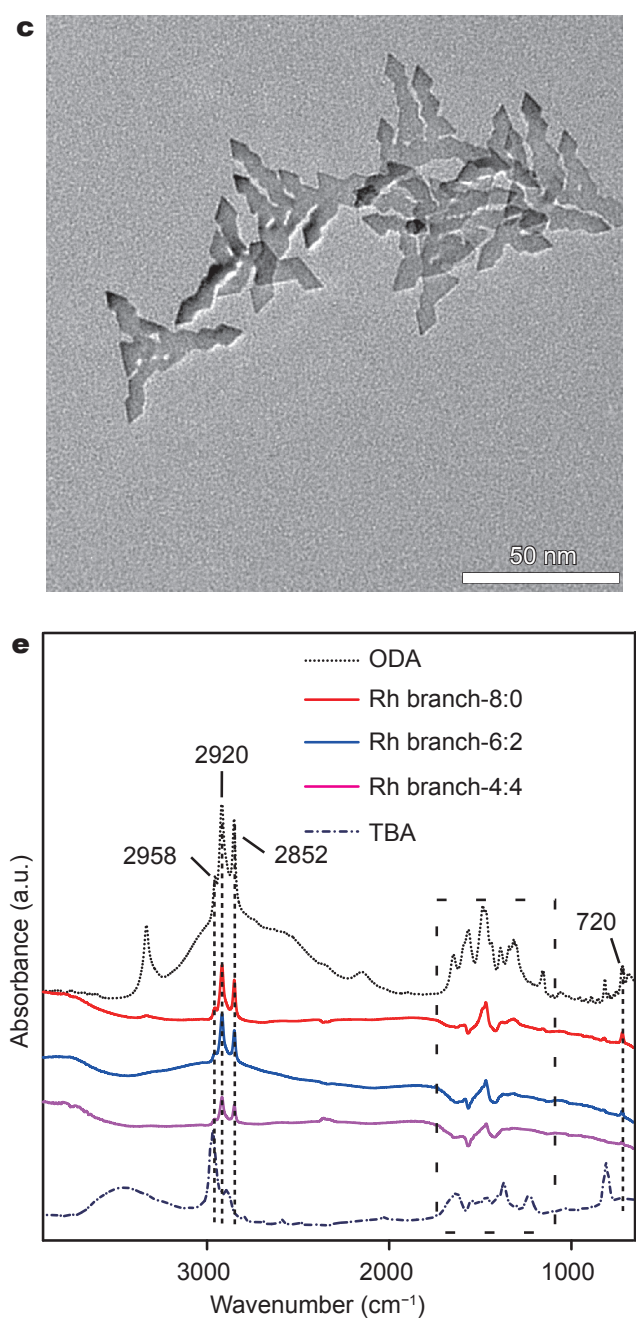

b

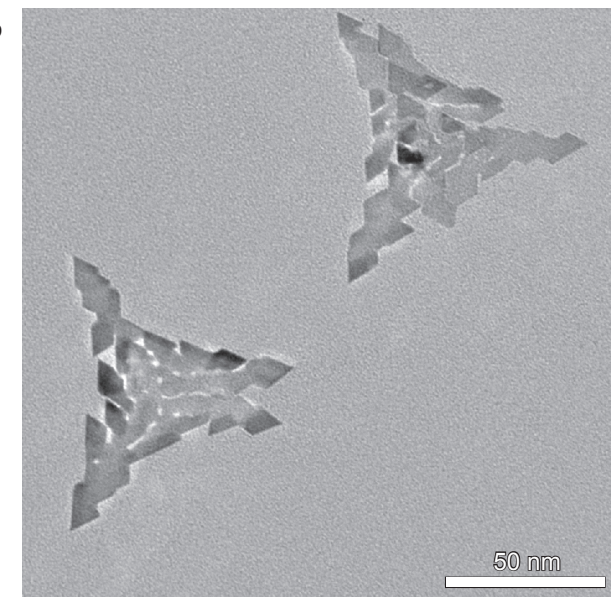

d
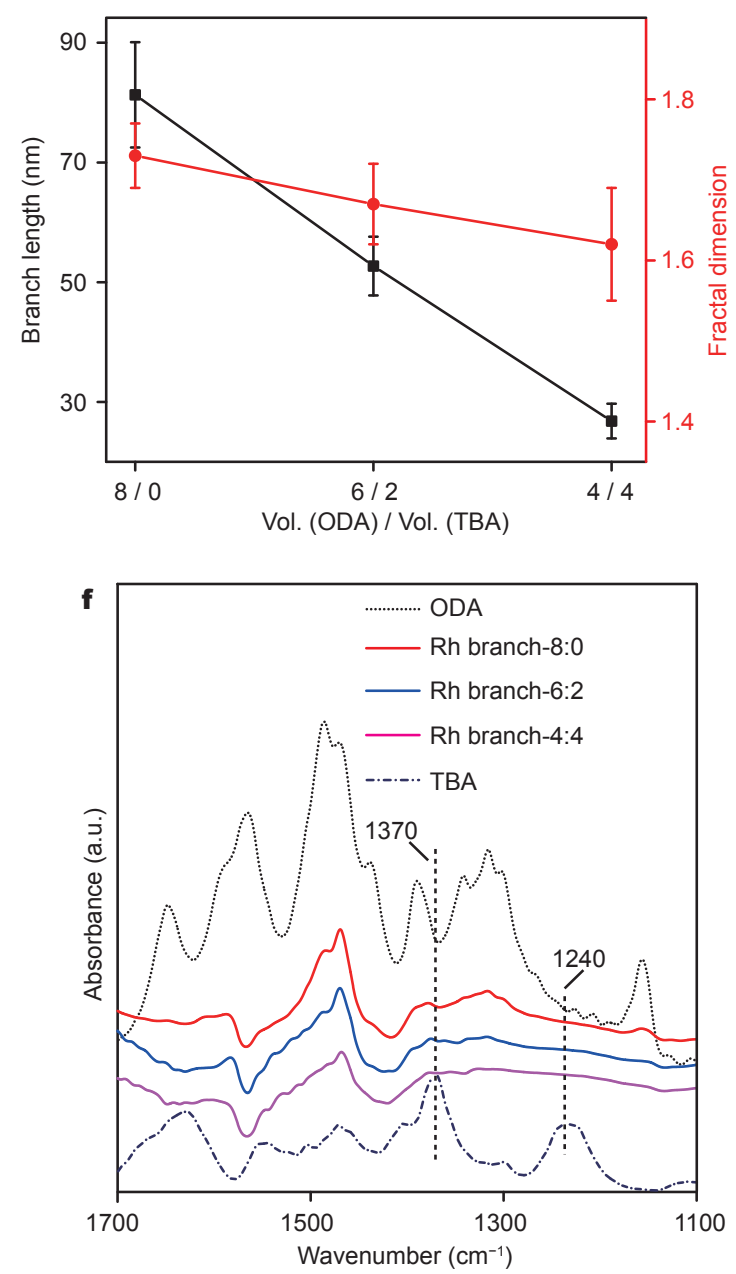

Figure 3 Representative TEM images of the products obtained at different volume ratios of ODA and TBA: (a) $8 \mathrm{~mL}: 0 \mathrm{~mL}$, (b) $6 \mathrm{~mL}: 2 \mathrm{~mL}$, (c) $4 \mathrm{~mL}$ : $4 \mathrm{~mL}$. (d) Statistical analysis of the corresponding length of branch arms and fractal dimension. (e) DRIFTS of the pure ODA, Rh branch-8:0, Rh branch-6:2, Rh branch-4:4, and pure TBA. (f) Zoom-in view of the area boxed in (e) range from 1700 to $1100 \mathrm{~cm}^{-1}$. 
favoring the formation of branched structures. When the amount of precursor was increased to $0.5 \mathrm{mg}(10 \%$ as compared to the standard synthesis), branch-like structures were observed (Fig. S7b). When increasing the amount of the precursor to $2 \mathrm{mg}$ ( $40 \%$ as compared to the standard synthesis), the driving force for the formation of branched NCs was further increased, and tri-pods with short arm (ca. $10 \mathrm{~nm}$ ) structures were obtained (Fig. S7c). However, at extremely high amounts of precursor (i.e., $30 \mathrm{mg}$, 6 times larger as compared to the standard synthesis), almost planar tripods with $50 \mathrm{~nm}$ arms were obtained (Fig. S7d). This may be attributed to the fact that an ultra-high concentration of precursor would induce numerous seeds in the nucleation stage. Thus, secondary triangles failed to grow from the planar tripods at these conditions. On basis of the two series of control experimental results described above, rational control of the growth unit diffusion process is essential for synthetizing our highly ordered hyperbranched $\mathrm{Rh}$ triangle nanoplates.

Owing to their large specific surface area and high density of low-coordinated edge and corner sites, highly branched fractal nanostructures usually show high catalytic activities towards numerous reactions [5,57,58]. Thus, the structural nature of the as-prepared hyperbranched $\mathrm{Rh}$ triangle nanoplates was firstly characterized taking advantage of the surface structure-dependent electrochemical cyclic voltammograms (CVs). Fig. S8 shows typical CVs in $0.1 \mathrm{~mol} \mathrm{~L}^{-1} \mathrm{HClO}_{4}$ for the as-prepared hyperbranched $\mathrm{Rh}$ triangle nanoplates as well as a state-of-the-art commercial Rh black catalyst (see TEM image in Fig. S9) that served as a reference for comparison. All the samples exhibited well-defined hydrogen and oxygen adsorption/desorption peaks. Importantly, these catalysts exhibited remarkably different current density in the oxygen adsorption/desorption region $(0.10-0.80 \mathrm{~V})$. It is well-known that the low-coordinated atoms such as steps, kinks, or structural defects can greatly enhance the oxygen adsorption, thus resulting in large oxygen adsorption/desorption currents [31,40]. All the hyperbranched $\mathrm{Rh}$ triangle nanoplates showed larger oxygen adsorption/desorption currents than the commercial $\mathrm{Rh}$ black, with Rh branch-6:2 exhibiting the largest current value. This result was in agreement with the density of edge/corner sites calculated for the different samples (Fig. S10). Thus, Rh branch-6:2 showed the largest density of edge/corner sites followed by Rh branch-4:4 and $\mathrm{Rh}$ branch-8:0.

In addition, electrochemical CO stripping experiments were conducted to determinate the electrochemically specific surface areas (ECSAs) of the catalysts. The elec- troactive surface areas of Rh branch-4:4, Rh branch-6:2, Rh branch-8:0, and commercial Rh black were 42.6, 43.7, 21.1, and $18.6 \mathrm{~m}^{2} \mathrm{~g}^{-1} \mathrm{Rh}$, respectively (Fig. 4a, Table S2). All the hyperbranched $\mathrm{Rh}$ triangle nanoplates possessed larger specific surface areas than the commercial Rh black catalyst. The Rh branch-6:2 showed the largest ECSA value (ca. 2.3 times that of the commercial Rh black catalyst). It is particularly worth mentioning that the onset potentials for CO stripping on Rh branch-4:4, Rh branch-6:2, and Rh branch-8:0 were lower than that of the commercial Rh black catalyst by 50,80 , and $20 \mathrm{mV}$, respectively (Fig. 4a, inset, see Table S2 for detailed onset potentials). According to previous studies, $\mathrm{Rh}$ surfaces with open structure are active towards the $\mathrm{CO}$ stripping reaction [59]. Clearly, the enhanced CO removal ability of these hyperbranched Rh triangle nanoplates should originate from the high densities of edge and corner sites of these materials.

The hyperbranched $\mathrm{Rh}$ triangle nanoplates having great CO removal ability and large ECSAs may find potential applications as fuel cell catalysts for the oxidation of small organic molecules, since electrocatalysts with outstanding CO tolerance and large electroactive surface areas are highly desired. Considering the excellent catalytic activity of $\mathrm{Rh}$ NCs towards the electrooxidation of ethanol in alkaline medium, we selected this model reaction to study the catalytic performance of the hyperbranched Rh triangle nanoplates. All oxidation currents were normalized to the ECSAs (obtained from the electric charges for $\mathrm{CO}$ stripping) and the corresponding loading mass. Fig. $4 \mathrm{~b}$ shows the CVs normalized against the loading mass of catalysts for the electro-oxidation of ethanol at room temperature $\left(\right.$ ca. $26^{\circ} \mathrm{C}$ ) in a $1.0 \mathrm{~mol} \mathrm{~L}^{-1}$ ethanol +1.0 mol L $\mathrm{L}^{-1} \mathrm{NaOH}$ solution. The electrocatalytic activity clearly increases in the order commercial Rh black $<\mathrm{Rh}$ branch-8:0 < Rh branch-4:4 < Rh branch-6:2. The Rh branch-6:2 exhibits the highest electrocatalytic activity (in the forward sweep) value (146.6 $\mathrm{A} \mathrm{mg}^{-1}$ ), which is $3.5,1.5$, and 5.2 times larger than those of $\mathrm{Rh}$ branch-8:0, $\mathrm{Rh}$ branch-4:4, and commercial Rh black catalysts, respectively. A similar tendency was observed when the oxidation currents were normalized to the ECSAs (Fig. 4c, Fig. S11, and Table S3). These results showed that the enhanced electrocatalytic activity towards the electro-oxidation of ethanol was highly related to the fractal structure, which contained large surface areas and high densities of active sites such as edge/corner atoms. Interestingly, our results reveal that the optimal catalytic activity can be tuned by varying the hierarchical ordering of the catalysts, where both the specific surface area and 

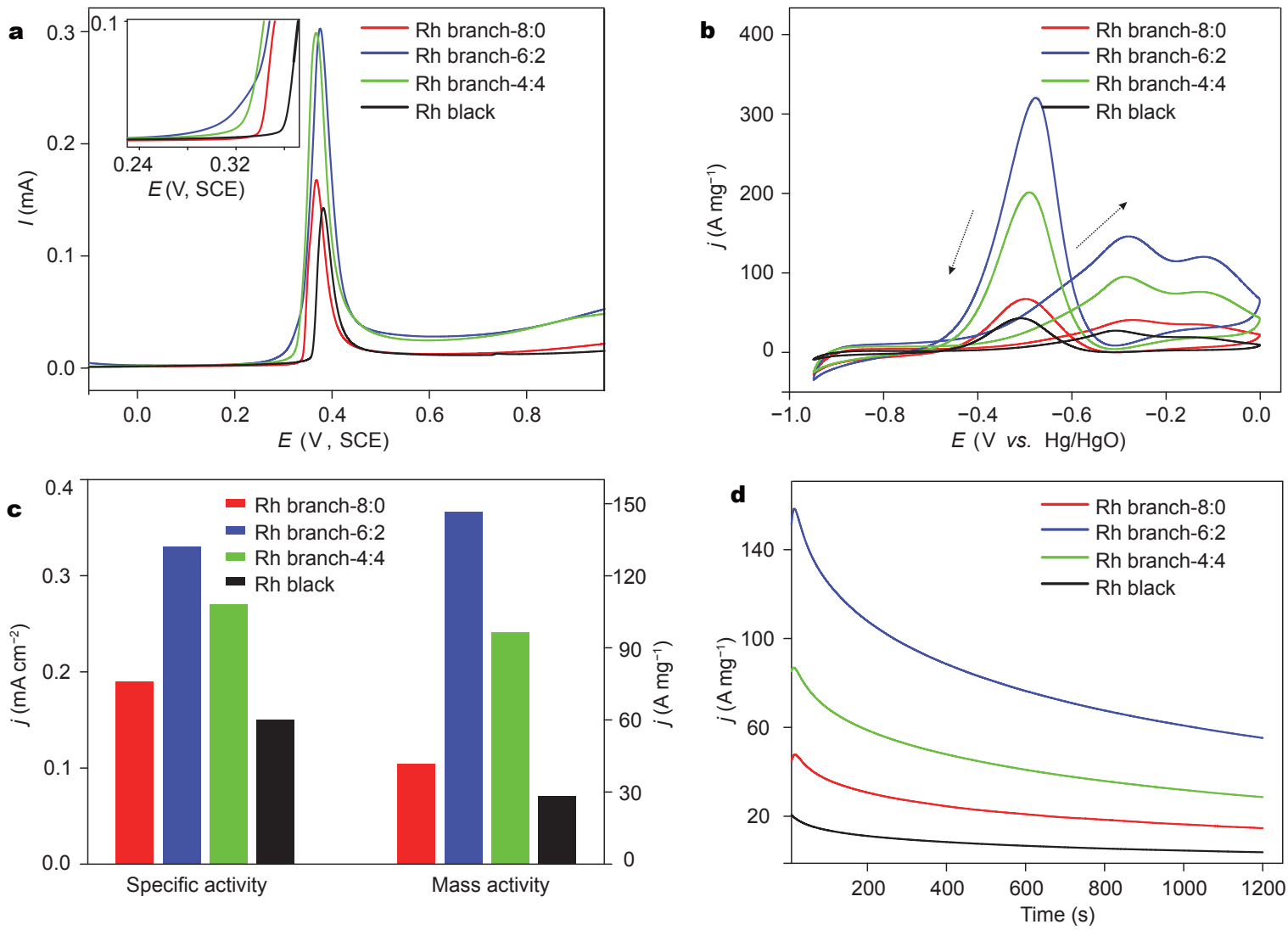

Figure 4 Electrochemical characterization of as-synthesized Rh NCs and commercial Rh black catalyst. (a) CO-stripping voltammetry in 0.1 mol L ${ }^{-1}$ $\mathrm{HClO}_{4}$ (scan rate: $20 \mathrm{mV} \mathrm{s}^{-1}$ ), (b) CVs of ethanol oxidation in $1.0 \mathrm{~mol} \mathrm{~L}^{-1}$ ethanol $+1.0 \mathrm{~mol} \mathrm{~L}^{-1} \mathrm{NaOH}$ solution (the arrows indicate the direction of the scan, scan rate: $50 \mathrm{mV} \mathrm{s}^{-1}$ ), all oxidation currents were normalized to the corresponding loaded mass on the working electrodes. (c) Comparison of the specific areas and mass activities of different catalysts. (d) Current-time curves for ethanol oxidation at $-0.35 \mathrm{~V}(v s$. $\mathrm{Hg} / \mathrm{HgO})$.

the density of edge and corner sites can be simultaneously optimized. Moreover, the hyperbranched Rh triangle nanoplates exhibited a good electrocatalytic stability. As shown in Fig. 4d, all the hyperbranched Rh triangle nanoplates displayed higher catalytic activities than the commercial $\mathrm{Rh}$ black catalyst over the entire measurement range. The remarkable stability of these hyperbranched Rh triangle nanoplates was further supported by the nearly unchanged morphology of the materials after the harsh electrochemical tests (Fig. S12). Additionally, the morphology of these hyperbranched Rh triangle nanoplates remained unchanged even after 6 months at room temperature and under atmospheric environment (Fig. S13).

Apart from being a high-performance electrocatalyst for fuel cells, $\mathrm{Rh}$ is also widely used as catalyst for hydrogenation reactions in the fine chemical industry. Edge and corner atoms with low coordination number have been demonstrated to be active in the liquid-phase hy- drogenation of styrene to ethylbenzene [60,61]. Therefore, we further selected the hydrogenation of styrene as a model test reaction to characterize the catalytic activity of our materials. Fig. 5 displays a comparison of the conversion of styrene to ethylbenzene as a function of the reaction time for the different $\mathrm{Rh}$ catalysts. Again, the best performance was achieved by Rh branch-6:2, followed by Rh branch-4:4, Rh branch-8:0, and commercial Rh black. The order of the catalytic hydrogenation activities followed the trend of the specific surface areas and the density of edge/corner sites. Likewise, in this model hydrogenation reaction, the large specific surface areas and high-densities of low-coordination edge/corner sites on the hyperbranched $\mathrm{Rh}$ triangle nanoplates prepared herein were essential for their significantly enhanced catalytic activity.

\section{CONCLUSIONS}

In summary, we firstly demonstrated that a single-crys- 

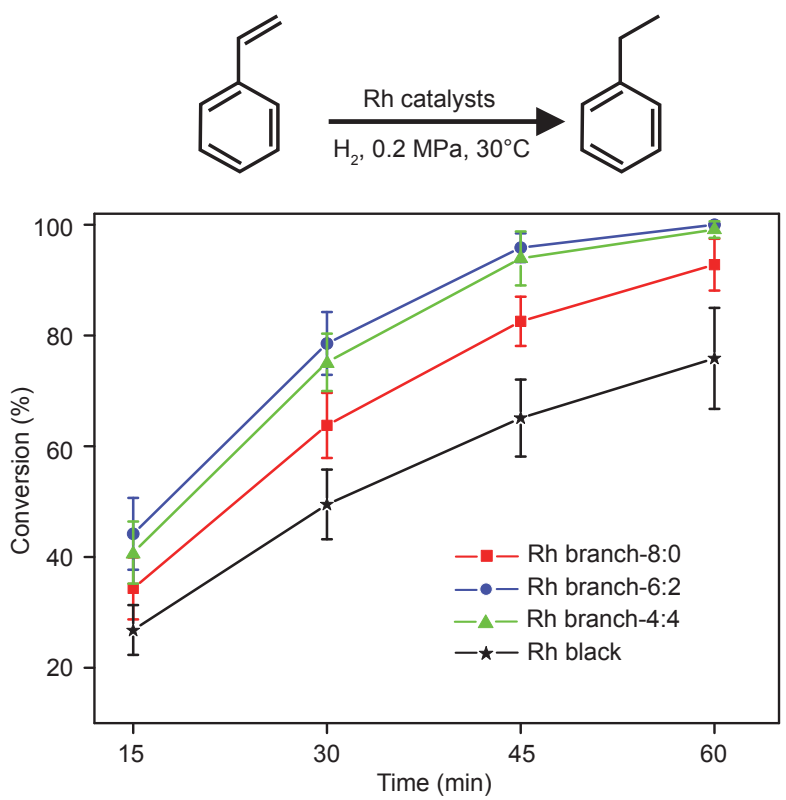

Figure 5 Catalytic hydrogenation ability of styrene by hyperbranched $\mathrm{Rh}$ NCs and commercial Rh black. Mole ratio between Rh catalyst and styrene was 1:500, $\mathrm{H}_{2}$ pressure: $0.2 \mathrm{MPa}$.

talline fractal nanostructure of $\mathrm{Rh}$ with well-defined shape and surface can be obtained by reducing $\mathrm{RhCl}_{3}$ with ODA without any surfactant or template. Such hyperbranched $\mathrm{Rh}$ triangle nanoplates have unique structure features, which are thin, threefold symmetric, and highly branched shape. The large steric barrier of ODA plays a vital role in the formation of hyperbranched structure. And the hierarchical ordering of the hyperbranched structure can be roughly controlled by intentionally introducing TBA. More importantly, the as-prepared hyperbranched $\mathrm{Rh}$ nanoplates possess large specific surface area as well as high-density of low-coordination edge and corner sites, which result in the outstanding catalytic activity towards the electrooxidation of ethanol and the hydrogenation of styrene. Besides, they exhibit great stability in hash electrochemical condition and atmospheric environment. We believe this work advances our understanding of the formation of fractal nanostructures, and would stimulate the exploration of other noble metal NCs with similar structures with their advantages in various applications.

Received 9 June 2017; accepted 11 July 2017; published online 8 August 2017

1 Gu J, Zhang YW, Tao FF. Shape control of bimetallic nanocatalysts through well-designed colloidal chemistry approaches. Chem Soc Rev, 2012, 41: 8050-8065

2 Jiang ZY, Kuang Q, Xie ZX, et al. Syntheses and properties of micro/nanostructured crystallites with high-energy surfaces. Adv Funct Mater, 2010, 20: 3634-3645

3 Wu Y, Wang D, Li Y. Understanding of the major reactions in solution synthesis of functional nanomaterials. Sci China Mater, 2016, 59: 938-996

4 Zhang L, Niu W, Xu G. Synthesis and applications of noble metal nanocrystals with high-energy facets. Nano Today, 2012, 7: 586605

5 Jia Y, Cao Z, Chen Q, et al. Synthesis of composition-tunable octahedral Pt-Cu alloy nanocrystals by controlling reduction kinetics of metal precursors. Sci Bull, 2015, 60: 1002-1008

6 Fu QQ, Li HH, Ma SY, et al. A mixed-solvent route to unique $\mathrm{PtAuCu}$ ternary nanotubes templated from $\mathrm{Cu}$ nanowires as efficient dual electrocatalysts. Sci China Mater, 2016, 59: 112-121

7 Chen Q, Jia Y, Xie S, et al. Well-faceted noble-metal nanocrystals with nonconvex polyhedral shapes. Chem Soc Rev, 2016, 45: 32073220

8 Feng Q, Wang W, Cheong WC, et al. Synthesis of palladium and palladium sulfide nanocrystals via thermolysis of a Pd-thiolate cluster. Sci China Mater, 2015, 58: 936-943

9 Chen X, Shi S, Wei J, et al. Two-dimensional Pd-based nanomaterials for bioapplications. Sci Bull, 2017, 62: 579-588

$10 \mathrm{Wu}$ J, Pan YT, Su D, et al. Ultrathin and stable AgAu alloy nanowires. Sci China Mater, 2015, 58: 595-602

11 Zhang J, Kuang Q, Jiang Y, et al. Engineering high-energy surfaces of noble metal nanocrystals with enhanced catalytic performances. Nano Today, 2016, 11: 661-677

12 Haruta M. Size- and support-dependency in the catalysis of gold. Catal Today, 1997, 36: 153-166

13 Bratlie KM, Lee H, Komvopoulos K, et al. Platinum nanoparticle shape effects on benzene hydrogenation selectivity. Nano Lett, 2007, 7: 3097-3101

14 Li M, Zhao Z, Cheng T, et al. Ultrafine jagged platinum nanowires enable ultrahigh mass activity for the oxygen reduction reaction. Science, 2016, 354: 1414-1419

15 Chen C, Kang Y, Huo Z, et al. Highly crystalline multimetallic nanoframes with three-dimensional electrocatalytic surfaces. Science, 2014, 343: 1339-1343

16 Mandelbrot B. How long is the coast of britain? Statistical selfsimilarity and fractional dimension. Science, 1967, 156: 636-638

17 Nakayama K, Silverman GH. Serial and parallel processing of visual feature conjunctions. Nature, 1986, 320: 264-265

18 Witten TA, Cates ME. Tenuous structures from disorderly growth processes. Science, 1986, 232: 1607-1612

19 Ben-Jacob E, Garik P. The formation of patterns in non-equilibrium growth. Nature, 1990, 343: 523-530

20 Addison PS. Fractals and Chaos: An Illustrated Course. London: Institute of Physics Publishing, 1997

21 Chen S, Wang ZL, Ballato J, et al. Monopod, bipod, tripod, and tetrapod gold nanocrystals. J Am Chem Soc, 2003, 125: 1618616187

22 Wang S, Xin H. Fractal and dendritic growth of metallic Ag aggregated from different kinds of $\gamma$-irradiated solutions. J Phys Chem B, 2000, 104: 5681-5685

23 Maksimuk S, Teng X, Yang H. Planar tripods of platinum: formation and self-assembly. Phys Chem Chem Phys, 2006, 8: 46604663

24 Chen J, Herricks T, Xia Y. Polyol synthesis of platinum nanostructures: control of morphology through the manipulation of reduction kinetics. Angew Chem Int Ed, 2005, 44: 2589-2592 
Ma L, Wang C, Gong M, et al. Control over the branched structures of platinum nanocrystals for electrocatalytic applications. ACS Nano, 2012, 6: 9797-9806

26 Watt J, Young N, Haigh S, et al. Synthesis and structural characterization of branched palladium nanostructures. Adv Mater, 2009, 21: 2288-2293

27 Xiao JP, Xie Y, Tang R, et al. Novel ultrasonically assisted templated synthesis of palladium and silver dendritic nanostructures. Adv Mater, 2001, 13: 1887-1891

28 Shelef M, McCabe RW. Twenty-five years after introduction of automotive catalysts: what next? Catal Today, 2000, 62: 35-50

29 Chen MS, Cai Y, Yan Z, et al. Highly active surfaces for CO oxidation on Rh, Pd, and Pt. Surf Sci, 2007, 601: 5326-5331

30 Duan $\mathrm{H}$, Yan N, Yu R, et al. Ultrathin rhodium nanosheets. Nat Commun, 2014, 5: 3093

31 Yu NF, Tian N, Zhou ZY, et al. Electrochemical synthesis of tetrahexahedral rhodium nanocrystals with extraordinarily high surface energy and high electrocatalytic activity. Angew Chem Int Ed, 2014, 216

32 Xie S, Liu XY, Xia Y. Shape-controlled syntheses of rhodium nanocrystals for the enhancement of their catalytic properties. Nano Res, 2015, 8: 82-96

33 Hoefelmeyer JD, Niesz K, Somorjai GA, et al. Radial anisotropic growth of rhodium nanoparticles. Nano Lett, 2005, 5: 435-438

34 Zettsu N, McLellan JM, Wiley B, et al. Synthesis, stability, and surface plasmonic properties of rhodium multipods, and their use as substrates for surface-enhanced raman scattering. Angew Chem Int Ed, 2006, 45: 1288-1292

35 Zhang Y, Grass ME, Kuhn JN, et al. Highly selective synthesis of catalytically active monodisperse rhodium nanocubes. J Am Chem Soc, 2008, 130: 5868-5869

36 Zhang H, Xia X, Li W, et al. Facile synthesis of five-fold twinned, starfish-like rhodium nanocrystals by eliminating oxidative etching with a chloride-free precursor. Angew Chim Int Ed, 2010, 49: 5296-5300

37 Biacchi AJ, Schaak RE. The solvent matters: kinetic versus thermodynamic shape control in the polyol synthesis of rhodium nanoparticles. ACS Nano, 2011, 5: 8089-8099

38 Zhang H, Li W, Jin M, et al. Controlling the morphology of rhodium nanocrystals by manipulating the growth kinetics with a syringe pump. Nano Lett, 2011, 11: 898-903

39 Xie S, Zhang H, Lu N, et al. Synthesis of rhodium concave tetrahedrons by collectively manipulating the reduction kinetics, facetselective capping, and surface diffusion. Nano Lett, 2013, 13: 62626268

40 Huang X, Zhao Z, Chen Y, et al. High density catalytic hot spots in ultrafine wavy nanowires. Nano Lett, 2014, 14: 3887-3894

41 Zhang SH, Jiang ZY, Xie ZX, et al. Growth of silver nanowires from solutions: a cyclic penta-twinned-crystal growth mechanism. J Phys Chem B, 2005, 109: 9416-9421

42 Liebovitch LS, Toth T. A fast algorithm to determine fractal dimensions by box counting. Phys Lett A, 1989, 141: 386-390

43 Lu L, Kobayashi A, Kikkawa Y, et al. Oriented attachment-based assembly of dendritic silver nanostructures at room temperature. J Phys Chem B, 2006, 110: 23234-23241

44 Fang J, You H, Kong P, et al. Dendritic silver nanostructure growth and evolution in replacement reaction. Cryst Growth Des, 2007, 7: 864-867

45 Zhang Q, Liu SJ, Yu SH. Recent advances in oriented attachment growth and synthesis of functional materials: concept, evidence, mechanism, and future. J Mater Chem, 2009, 19: 191-207

46 Witten Jr. TA, Sander LM. Diffusion-limited aggregation, a kinetic critical phenomenon. Phys Rev Lett, 1981, 47: 1400-1403

47 Witten TA, Sander LM. Diffusion-limited aggregation. Phys Rev B, 1983, 27: 5686-5697

48 Ming NB, Wang M, Peng RW. Nucleation-limited aggregation in fractal growth. Phys Rev E, 1993, 48: 621-624

49 Wang M, Liu XY, Strom CS, et al. Fractal aggregations at low driving force with strong anisotropy. Phys Rev Lett, 1998, 80: 3089-3092

50 Liu XY, Wang M, Li DW, et al. Nucleation-limited aggregation of crystallites in fractal growth. J Cryst Growth, 2000, 208: 687-695

51 Miller CC. The Stokes-Einstein law for diffusion in solution. Proc R Soc A-Math Phys Eng Sci, 1924, 106: 724-749

52 Murray CB, Kagan CR, Bawendi MG. Self-organization of CdSe nanocrystallites into three-dimensional quantum dot superlattices. Science, 1995, 270: 1335-1338

53 Badia A, Singh S, Demers L, et al. Self-assembled monolayers on gold nanoparticles. Chem Eur J, 1996, 2: 359-363

54 Gebben B, van den Berg HWA, Bargeman D, et al. Intramolecular crosslinking of poly(vinyl alcohol). Polymer, 1985, 26: 1737-1740

55 Yang Y, Qin H, Jiang M, et al. Entropic ligands for nanocrystals: from unexpected solution properties to outstanding processability. Nano Lett, 2016, 16: 2133-2138

56 Socrates G. Infrared and Raman Characteristic Group Frequencies: Tables and Charts. Chichester: John Wiley \& Sons, 2001

57 Lim B, Xia Y. Metal nanocrystals with highly branched morphologies. Angew Chem Int Ed, 2011, 50: 76-85

58 Ye E, Regulacio MD, Zhang SY, et al. Anisotropically branched metal nanostructures. Chem Soc Rev, 2015, 44: 6001-6017

59 Housmans THM, Koper MTM. CO oxidation on stepped $\mathrm{Rh}[n(111) \times(111)]$ single crystal electrodes: anion effects on CO surface mobility. Electrochem Commun, 2005, 7: 581-588

60 Veisz B, Király Z, Tóth L, et al. Catalytic probe of the surface statistics of palladium crystallites deposited on montmorillonite. Chem Mater, 2002, 14: 2882-2888

61 Király Z, Veisz B, Mastalir Á. $\mathrm{CS}_{2}$ poisoning of size-selective cubooctahedral $\mathrm{Pd}$ particles in styrene hydrogenation. Catal Lett, 2004, 95: 57-59

Acknowledgements This work was supported by the National Basic Research Program of China (2015CB932301), the National Natural Science Foundation of China (21333008, 21671163, 21603178 and J1310024), and China Postdoctoral Science Foundation (2016M602066 and 2017T100468). We particularly appreciate Shilong Liu at the University of Science and Technology of China for the analysis of fractal dimensions.

Author contributions Kuang Q and Xie Z proposed the research direction and guided the whole project. Zhang J designed and performed the experiments and wrote the manuscript; Chen M, Chen J, Li H and Wang S helped draft the manuscript and analyze the data. All authors contributed to the general discussion and reviewed the manuscript.

Conflict of interset The authors declare that they have no conflict of interest.

Supplementary information Experimental details are available in the online version of the paper. 

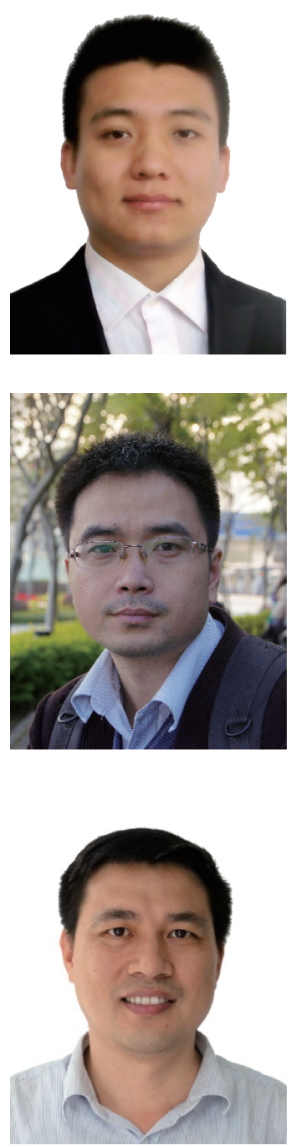

Jiawei Zhang received his BSc degree from Jinan University in 2009. Then he joined the Department of Chemistry at Xiamen University as a graduate and obtained his $\mathrm{PhD}$ degree under the supervision of Prof. Zhaoxiong Xie in 2015. He is currently working as a postdoctoral research fellow in Prof. Xie's group. His research focuses on the controlled synthesis of noble metal nanocrystals, and their applications in catalysis and fuel cells.

Qin Kuang received his BSc degree in 2001 and PhD degree in 2008 from Xiamen University, China. Thereafter, he joined the Department of Chemistry as an assistant professor in Xiamen University and was promoted to associate professor in 2009. His current research focuses on the surface/interface engineering of inorganic functional nanomaterials and their applications in energy and environmental fields.

Zhaoxiong Xie received his BSc degree (1987) in chemistry, MSc degree (1990), and PhD degree (1995) in physical chemistry from Xiamen University, China. He worked as a postdoctoral fellow at the Centre d'Etudes de Saclay in France from 1997 to 1998. Since 2002, he has been a professor of physical chemistry at Xiamen University. His current research is focused on the surface/interface chemistry of inorganic nanomaterials.

\section{超支化Rh单晶纳米薄片的合成及其催化性能研究}

张嘉伟, 陈梅珊, 陈佳显, 李慧齐, 王苏恒, 匡勤, 曹振明, 谢兆雄 ${ }^{*}$

摘要 贵金属纳米晶的催化性质与其结构密切相关. 比表面积越大、配位不饱和的边角原子密度越高, 贵金属纳米晶在催化反应过程中表现 出的性能往往越优异. 相比于常见的具有完整几何形貌的贵金属纳米晶, 具有多重分级结构特征的超支化贵金属纳米晶拥有更大的比表面积 以及更丰富的配位不饱和的活性位点, 因此被认为是一种潜在的性能优异的催化剂. 但这种具有多重分级结构特征的超支化贵金属纳米晶无 论是在生长机理研究还是可控制备上都还存在巨大的挑战. 本文通过简单的湿化学法成功制备出一种具有多重分级结构特征的超支化 $\mathrm{Rh}$ 纳 米薄片. 该产物由三角形纳米片在扩散限制条件下分级生长形成, 整体呈现三次对称性的单晶特征. 研究表明, 该纳米结构不仅具有出色的结 构稳定性, 而且其生长级数可通过反应溶剂比例的简单调节进行调控, 从而实现Rh纳米薄片比表面积和位于边/角活性位点的原子比例的调 控. 由于拥有更大的比表面积以及更为丰富的配位不饱和的活性位点, 这种具有多重分级结构的超支化Rh纳米薄片在乙醇电催化氧化和苯乙 烯催化加氢催化反应中展现出了比目前主流商业催化剂Rh黑更为优异的催化活性. 